\title{
BMJ Open Cohort profile: the Singapore diabetic cohort study
}

\author{
Miyang Luo, ${ }^{1}$ Linda Wei Lin Tan, ${ }^{1}$ Xueling Sim, ${ }^{1}$ Milly Khiam Hoon Ng, \\ Rob Van Dam, ${ }^{1}$ E Shyong Tai, ${ }^{1,2}$ Kee Seng Chia, ${ }^{1}$ Wern Ee Tang, ${ }^{3}$ Darren EJ Seah, ${ }^{3}$ \\ Kavita Venkataraman
}

To cite: Luo M, Tan LWL, Sim X, et al. Cohort profile: the Singapore diabetic cohort study. BMJ Open 2020;10:e036443. doi:10.1136/ bmjopen-2019-036443

- Prepublication history and additional material for this paper are available online. To view these files, please visit the journal online (http://dx.doi. org/10.1136/bmjopen-2019036443).

Received 18 December 2019 Revised 21 March 2020 Accepted 17 April 2020

Check for updates

(C) Author(s) (or their employer(s)) 2020. Re-use permitted under CC BY-NC. No commercial re-use. See rights and permissions. Published by BMJ.

${ }^{1}$ Saw Swee Hock School of Public Health, National University of Singapore and National University Health System, Singapore

${ }^{2}$ Division of Endocrinology, National University Hospital, Singapore

${ }^{3}$ National Healthcare Group Polyclinics, Singapore

Correspondence to Dr Kavita Venkataraman; kavita_v@nus.edu.sg

\section{ABSTRACT}

Purpose The diabetic cohort (DC) was set up to study the determinants of complications in individuals with type 2 diabetes and examine the role of genetic, physiological and lifestyle factors in the development of complications in these individuals.

Participants A total of 14033 adult participants with type 2 diabetes were recruited from multiple public sector polyclinics and hospital outpatient clinics in Singapore between November 2004 and November 2010. The first round of follow-up was conducted for 4131 participants between 2012 and 2016; the second round of followup started in 2016 and is expected to end in 2021. A questionnaire survey, physical assessments, blood and urine sample collection were conducted at recruitment and each follow-up visit. The data set also includes genetic data and linkage to medical and administrative records for recruited participants

Findings to date Data from the cohort have been used to identify determinants of diabetes and related complications. The longitudinal data of medical records have been used to analyse diabetes control over time and its related outcomes. The cohort has also contributed to the identification of genetic loci associated with type 2 diabetes and diabetic kidney disease in collaboration with other large cohort studies. About 25 scientific papers based on the DC data have been published up to May 2019.

Future plans The rich data in DC can be used for various types of research to study disease-related complications in patients with type 2 diabetes. We plan to further investigate disease progression and new biomarkers for common diabetic complications, including diabetic kidney disease and diabetic neuropathy.

\section{INTRODUCTION}

Diabetes mellitus is a major public health problem that has reached epidemic proportions globally. The International Diabetes Federation has estimated that there were more than 400 million individuals with diabetes worldwide in 2017, which is projected to increase over 600 million by $2045,{ }^{12}$ with around $90 \%$ having type 2 diabetes. Diabetes also causes vascular damage in multiple organ systems, leading to increased risk of cardiovascular diseases, chronic kidney disease, retinopathy and lower limb amputations. The

\section{Strengths and limitations of this study}

- The cohort focuses on diabetes, one of the biggest public health challenges in this century, with a relatively large pool of multiethnic participants comprising Chinese, Malay and Indians, and follow-up duration of more than a decade.

- This cohort has collected a variety of data on sociodemographics, lifestyle, health-related quality of life, anthropometric and other physical measurements, biochemical characteristics and genetic profiles through questionnaires, physical assessment, collection of biological samples and linkage to medical and administrative records.

- Diabetic cohort is a prevalence cohort, which recruited participants with varying durations of disease, and potentially at different stages of the natural history of disease.

- This cohort may be limited by the low rate of active follow-up of participants, which has been substantially overcome through the linkage with medical records and disease registries.

global burden of such complications is huge, with diabetes now a leading cause for endstage renal disease, blindness and disability. ${ }^{3-7}$ Therefore, diabetes and diabetes-related complications contribute substantially to the global burden of disease, in terms of morbidity, ${ }^{8}$ mortality, ${ }^{9}$ reduced quality of life ${ }^{1011}$ and economic cost. ${ }^{12}$

Rates of type 2 diabetes and related complications vary significantly across countries and regions. In particular, Asians are not only at higher risk for type 2 diabetes at lower levels of obesity and younger ages but also at increased risk of adverse outcomes. ${ }^{13}{ }^{14}$ In Singapore, the prevalence of diabetes has been rising, with prevalences of $8.3 \%$ and $8.6 \%$ being reported, using fasting plasma glucose measurements only, in the consecutive National Health Surveys in 2010 and 2017, respectively. ${ }^{15}$ Incidence rates of serious diabetes-related complications like end-stage renal disease and lower extremity 
amputations are much higher in Singapore as compared with other high-income countries. ${ }^{616}$

The diabetic cohort (DC), therefore, was set up to study the determinants of complications in individuals with type 2 diabetes and examine the role of genetic, physiological and lifestyle factors in the development of complications in these individuals. As a disease cohort in multiethnic Singapore, the DC provides the opportunity to examine intra-Asian variations in risk of complications and downstream outcomes and the role of the factors mentioned above in any differential risk.

Through the DC, we hope to provide a more complete understanding of the etiopathogenesis of type 2 diabetes and its related complications in Asia, with the long-term objective of improving care and outcomes in individuals with type 2 diabetes.

\section{Cohort description}

The DC is a part of the Singapore Population Health Studies in the Saw Swee Hock School of Public Health, National University of Singapore. It is a closed cohort with a total of 14033 adults with diabetes enrolled during two recruitment phases in 2004-2006 and 2006-2010 from multiple public sector polyclinics and hospital outpatient clinics. In phase 1, 5324 participants were recruited from Clementi and Toa Payoh Polyclinics, Tan Tock Seng Hospital and National University Hospital in Singapore. An additional set of 8709 participants were recruited from Choa Chu Kang, Jurong, Yishun and Pasir Ris Polyclinics, as well as Changi General Hospital, and Alexandra Hospital in phase 2. Inclusion criteria for the DC were adult Singaporeans and permanent residents (aged 21 years and above) with physician-diagnosed type 2 diabetes. Subjects with mental illness, clinically obvious non-diabetic kidney disease (such as polycystic kidney disease), type 1 diabetes or diabetes mellitus resulting from endocrinopathies were excluded.

\section{PATIENT RECRUITMENT}

Recruitment was conducted in the following ways: (1) patients were identified by their attending physicians and referred to a research nurse or identified by a research nurse based on review of medical records and approached directly for recruitment at the clinic or (2) patients were approached by a trained researcher at the waiting area of the clinic for assessment of eligibility and recruitment. Written informed consent was obtained for (1) participation in the study, (2) extraction of information from case notes, (3) linkage to national disease registries and medical records, (4) storage of biospecimens for future research and (5) recontact. After informed consent, each participant completed an interviewer-administered questionnaire and underwent anthropometric assessments by a trained research nurse. Blood and spot urine specimens were collected at the time of recruitment and stored for future analysis. If the patient had already provided a blood specimen for the quarterly diabetes monitoring tests to the clinic prior to the providing consent, the blood specimen would be collected at the patients' next quarterly diabetes monitoring visit. Participants' medical record data of the past 5 years were extracted from the site of recruitment by the trained research nurses after enrolment.

\section{Patient and public involvement}

Patients or the public were not involved in the design, conduct, reporting or dissemination plans of our research.

\section{FOLLOW-UP INFORMATION}

After the baseline recruitment in 2004 and 2010, 410 participants of the DC were selected for a pilot follow-up in 2010 to study the associations between the glycaemic index of their diet with glycaemic control and risk factors for cardiovascular disease. Following the pilot, the first wave of in-person follow-up with the cohort participants began in 2012 and ended in 2016. Of the 14033 baseline participants, 4677 (33.3\%) were not contactable (ie, contact details changed and no updated information available, frequently travelling, or access to household denied and unable to contact despite six attempts at household visitation) and $1058(7.5 \%)$ were confirmed to have been lost to follow-up (ie, deceased, migrated, declined consent for follow-up at baseline, lost mental competence to give consent to continue the research, institutionalised or physically unfit to participate). Of the 8298 contactable participants, 4131 (29.4\% of DC and $49.8 \%$ of contactable participants) agreed to participate in the follow-up survey. A second wave of in-person follow-up of the DC has been started in 2016 and is expected to be completed in 2021.

\section{DATA COLLECTION}

Table 1 summarises the information collected from DC participants at baseline and follow-up assessment. At recruitment, participants completed an intervieweradministered questionnaire, which took approximately 15-20 min. Questionnaires were available in English, and interviewers provided additional explanation and translation when necessary in other languages common to both the participant and the interviewer. Participants were interviewed about their demographic characteristics, smoking behaviour and personal and family medical history. Height and weight were based on the last recorded values in the medical record file of the participants which would have been measured on the same day or up to 3 months earlier. Waist circumference was measured at the level of the mid-point between the last rib and the iliac crest, with the participant in light expiration following WHO standards. Hip circumference was measured at the level of the greater trochanter of the femur. ${ }^{17}$

Follow-up of the DC was conducted in tandem with the follow-up of the Singapore Multiethnic Cohort using the same study protocol. ${ }^{18}$ During the first follow-up 
Table 1 Summary of variables collected or derived from the cohort

\section{Variables}

Baseline First follow-up Second follow-up

Questionnaire

Demographics

Tobacco use

Environmental tobacco smoke

Alcohol consumption

Diet (FFQ)

Physical activity

Sleep quality (PSQI)

Medication use

Medical history

History of diabetes complications

Women's health

Skin health

Family history

Health-related quality of life (SF-12/SF-36/EQ-5D)

Stress

Kessler Psychological Distress Scale (K10)

Cognitive test (MMSE for age 40 years and older)

Workability

ADL (for age 65 years and older)

Instrumental ADL (for age 65 years and older)

Physical examination

\begin{tabular}{|c|c|c|c|}
\hline Height & $\checkmark$ & $\checkmark$ & $\checkmark$ \\
\hline Weight & $\checkmark$ & $\checkmark$ & $\checkmark$ \\
\hline Waist circumference & $\checkmark$ & $\checkmark$ & $\checkmark$ \\
\hline Blood pressure & & $\checkmark$ & $\checkmark$ \\
\hline Central aortic blood pressure & & $\checkmark$ & \\
\hline Skinfold thickness & & $\checkmark$ & \\
\hline Resting ECG & & $\checkmark$ & $\checkmark$ \\
\hline $\begin{array}{l}\text { Assessment of foot sensory function by monofilament and } \\
\text { neurothesiometer }\end{array}$ & & $\checkmark$ & $\checkmark$ \\
\hline Spirometry (for ages 35-80) & & & $\checkmark$ \\
\hline \multicolumn{4}{|l|}{ Medical records } \\
\hline Diabetes profile and treatment & $\checkmark$ & $\checkmark$ & \\
\hline Blood pressure profile and treatment & $\checkmark$ & $\checkmark$ & \\
\hline Lipid profile and treatment & $\checkmark$ & $\checkmark$ & \\
\hline Complications of kidney, eye and macrovascular systems & $\checkmark$ & $\checkmark$ & \\
\hline
\end{tabular}

Continued 
Table 1 Continued

\begin{tabular}{lll}
\hline Variables & Baseline & First follow-up Second follow-up \\
\hline Urine protein, glucose, ketone and blood (semiquantitative) & $\checkmark$ & $\checkmark$ \\
Urine albumin (semiquantitative) & $\checkmark$ & $\checkmark$ \\
High sensitive C-reactive protein & $\checkmark$ & $\checkmark$ \\
Cortisol & $\checkmark$ & $\checkmark$ \\
Serum creatinine & $\checkmark$ \\
Fasting glucose & $\checkmark$ \\
Blood lipids & $\checkmark$ \\
Haemoglobin A1c & $\checkmark$ \\
Interleukin-6 & $\checkmark$ \\
Interleukin-1 receptor antagonist & $\checkmark$ \\
\hline
\end{tabular}

$A D L$, activities of daily living; EQ-5D, EuroQol quality of life 5 dimensions; FFQ, food frequency questionnaire; MMSE, mini-mental state examination; PSQI, Pittsburgh sleep quality index; SF-12/SF-36, short form health survey - 12 items/ 36 items.

assessment, participants completed a more comprehensive questionnaire that included additional information on environmental tobacco smoke, alcohol consumption, diet, physical activity, medication use, women's health, health-related quality of life, stress, distress (Kessler Psychological Distress Scale (K10)) and cognition (minimental state examination). This was followed by a physical examination to measure height, weight, waist circumference, hip circumference, brachial and ankle blood pressure, central aortic blood pressure, skinfold thickness, resting ECG, hand grip strength and foot sensory function using monofilament and neurothesiometer. Systolic and diastolic blood pressures were measured using an automated digital monitor (Dinamap Carescape V100, General Electronic). A sphygmomanometer (Accoson, UK) was used for participants with blood pressures beyond the range of the digital monitor. Two readings were recorded for each participant, with a third reading if the difference between the first two readings exceeded $10 \mathrm{~mm} \mathrm{Hg}$ for systolic or $5 \mathrm{~mm} \mathrm{Hg}$ for diastolic blood pressure, respectively. Central aortic systolic pressure and arterial pulse waveform were measured on the left arm with the participant seated and the left arm resting on a table at chest level using A-PULSE CASPro Lite (HealthSTATS, Singapore). Skinfold thickness was measured by a Holtain/Tanner skinfold calliper at the left triceps, left biceps, subscapular, supra-iliac and calf regions with the participant in a standing position. A resting electrocardiogram (10 leads) was obtained using the ECG-1350K (Nihon Kohden, Japan). A hand dynamometer (TAKEI A5401, Japan) was used for assessing hand grip strength with three readings recorded for each arm. Foot sensory function was assessed with the participant in supine position with bare feet and closed eyes. A $10 \mathrm{~g}$ (5.07) monofilament (Sensory Testing System, USA) was used to test light touch on five least calloused plantar sites per foot-the distal great toe, third toe, fifth toe and the first and fifth metatarsal heads. The number of sites that the participants could feel was recorded for each foot. A neurothesiometer (Horwell, UK) was used to assess foot proprioception. A vibration-emitting probe with gradually increased voltage was applied to the apex of the big toe and medial malleolus of both feet, and the voltage reading was recorded when the participants indicated verbally that they could feel the vibration.

During the second follow-up, assessments of workability and activities of daily living were also included in the questionnaire, and measurements of visual acuity, Timed-Upand-Go and spirometry have been added to the physical examination.

\section{Medical records extraction and data linkage}

After recruitment, relevant data were extracted from patients' medical case notes available at the site of recruitment. Extraction was restricted to a period of up to 5 years before recruitment and was performed by trained research nurses. Fields extracted included records of diagnosis of diabetes, haemoglobin A1c (HbAlc) levels, treatment regimens for diabetes, hypertension diagnosis, blood pressure levels, antihypertensive treatment, lipid values, usage of lipid-lowering agents, diagnosis of microalbuminuria, proteinuria and diabetic nephropathy; age at dialysis initiation, age at renal transplant, serum creatinine levels, urinalysis results, and diagnosis of diabetic retinopathy, cataract, ischaemic heart disease, stroke and limb amputation.

Additional comprehensive medical data on physical and laboratory investigations and medications for the period 2000-2015 were obtained by linkage with the electronic medical record system of the National Healthcare Group (NHG) Polyclinics for participants who ever visited the NHG polyclinics ( $\mathrm{n}=11721$ participants). Extracted data included blood test results (ie, measurements of fasting or random glucose, HbA1c, lipids, creatinine, haemoglobin, 
Table 2 Biosample availability for baseline and first followup

\begin{tabular}{lcc}
\hline Biosamples & Baseline* $^{*}$ & $\begin{array}{l}\text { First } \\
\text { follow-up * }\end{array}$ \\
\hline $\begin{array}{l}\text { Whole blood/whole blood } \\
\text { plasma }\end{array}$ & 7569 & 2255 \\
$\begin{array}{l}\text { DNA/buffy coat/buffy coat } \\
\text { DNA }\end{array}$ & 11901 & 2400 \\
$\begin{array}{l}\text { Plasma/plasma (sodium } \\
\text { citrate) }\end{array}$ & 9049 & 2414 \\
$\begin{array}{l}\text { Serum } \\
\text { Red blood cells }\end{array}$ & 8008 & 2040 \\
\hline Urine (buffered/normal) & 7123 & 372 \\
\hline
\end{tabular}

*Number of participants with at least one aliquot of sample available. Sample availability was updated on 11 March 2020.

urea and uric acid), physical measurements (ie, blood pressure, height and weight), urine test results (ie, albumin, albumin creatinine ratio, protein, protein creatinine ratio, creatinine, cells and formed elements), medication records, clinic visits records and attendance at diabetic food screening and retinal photography. Cohort data have also been linked with the disease registers maintained by the National Registry of Diseases Office to identify the incidence of acute myocardial infarction, stroke, end-stage renal disease, cancer and death.

\section{Biochemical analyses and biobanking}

Blood and urine samples were collected from consenting participants at baseline and follow-up. In the first phase of recruitment, approximately $10 \mathrm{~mL}$ of blood (fasting or random) and $50 \mathrm{~mL}$ of urine were obtained from each consented participant; in the second phase, $15 \mathrm{~mL}$ of blood and $20 \mathrm{~mL}$ of urine were taken. During follow-up, participants were asked to fast $8-12$ hour before their appointment, and up to $23 \mathrm{~mL}$ of blood and $6 \mathrm{~mL}$ of urine were stored. The samples were aliquoted and stored at $-80^{\circ} \mathrm{C}$. DNA, buffy coat, plasma, serum and red blood cells were extracted from blood samples and stored separately. The number of biosamples available is summarised in table 2.

Genome-wide array data are available for a subset of the DC cohort from a combination of Illumina genomewide genotyping arrays $(n=2010)^{19}$ and imputed to $1000 \mathrm{G}$ Phase 3 reference panels. In addition, whole exome sequence data are also available for some of the participants as part of the T2D-GENES (Type 2 Diabetes Genetic Exploration by Next-generation sequencing in multiEthnic Samples) Consortium. ${ }^{20} 21$

\section{PARTICIPANT CHARACTERISTICS}

The demographic profile of participants at recruitment is presented in table 3 . The mean age of participants was $59.7 \pm 10.7$ years and $50.8 \%$ were men. The ethnic composition was $59.3 \%$ Chinese, $22.7 \%$ Malay and $17.3 \%$ Indian.
Table 3 Participants characteristics at baseline $(n=14033)$

\begin{tabular}{lc}
\hline \multicolumn{1}{c}{$\mathbf{N}(\%)$} \\
\hline $\begin{array}{l}\text { Age at interview (in years), mean } \pm \\
\text { SD }\end{array}$ & $59.7(10.7)$ \\
$\begin{array}{l}\text { Duration of diabetes (years), median } \\
\text { (IQR) }\end{array}$ & $7.0(3.0-14.0)$ \\
\hline
\end{tabular}

(IQR)

\begin{tabular}{|c|c|}
\hline \multicolumn{2}{|l|}{ Gender } \\
\hline Male & $7134(50.8)$ \\
\hline Female & $6899(49.2)$ \\
\hline \multicolumn{2}{|l|}{ Ethnicity } \\
\hline Chinese & $8327(59.3)$ \\
\hline Malay & $3181(22.7)$ \\
\hline Indian & $2423(17.3)$ \\
\hline Others & $102(0.7)$ \\
\hline \multicolumn{2}{|l|}{ Marital status } \\
\hline Never married & $710(5.1)$ \\
\hline Currently married & $10779(76.9)$ \\
\hline Separated/divorced & $490(3.5)$ \\
\hline Widowed & $2047(14.6)$ \\
\hline \multicolumn{2}{|l|}{ Education status* } \\
\hline No formal qualification & $3647(26.0)$ \\
\hline Primary & $4614(32.9)$ \\
\hline Secondary & $3832(27.3)$ \\
\hline Vocational training/postsecondary & $1388(9.9)$ \\
\hline University and above & $546(3.9)$ \\
\hline \multicolumn{2}{|l|}{ Occupation status } \\
\hline Working & $5909(42.2)$ \\
\hline Homemaker & $4426(31.6)$ \\
\hline Retired & $3101(22.1)$ \\
\hline Unemployed & $574(4.1)$ \\
\hline \multicolumn{2}{|l|}{ Self-reported health conditions $(\%) \dagger$} \\
\hline Hypertension & $9608(68.5)$ \\
\hline Diabetic retinopathy & $1534(10.9)$ \\
\hline Diabetic kidney disease & $1268(9.0)$ \\
\hline \multicolumn{2}{|l|}{ Family history (\%)‡ } \\
\hline Hypertension & $6835(48.7)$ \\
\hline Diabetes & $10755(76.6)$ \\
\hline \multicolumn{2}{|l|}{ Smoking status (\%) } \\
\hline Never smoker & 10043 (71.6) \\
\hline Ex-smoker & $2370(16.9)$ \\
\hline Current smoker & $1619(11.5)$ \\
\hline
\end{tabular}

Numbers missing: duration of diabetes $(n=927)$, marital status $(n=7)$, educational status $(n=6)$, occupation status $(n=23)$ and smoking status $(n=1)$.

*Educational status: secondary education ('O'/'N' level), vocational training (attended Institute of Technical Education or obtained National Technical Certificate) and postsecondary education (' $A$ ' level, polytechnic/diploma).

†Participants were asked whether they had been diagnosed with hypertension, diabetic retinopathy or diabetic kidney disease by Western doctors.

‡Family history was defined as having a history of the condition in parents or siblings. 
Table 4 Disease profile of study participants at recruitment and the latest visit in medical records

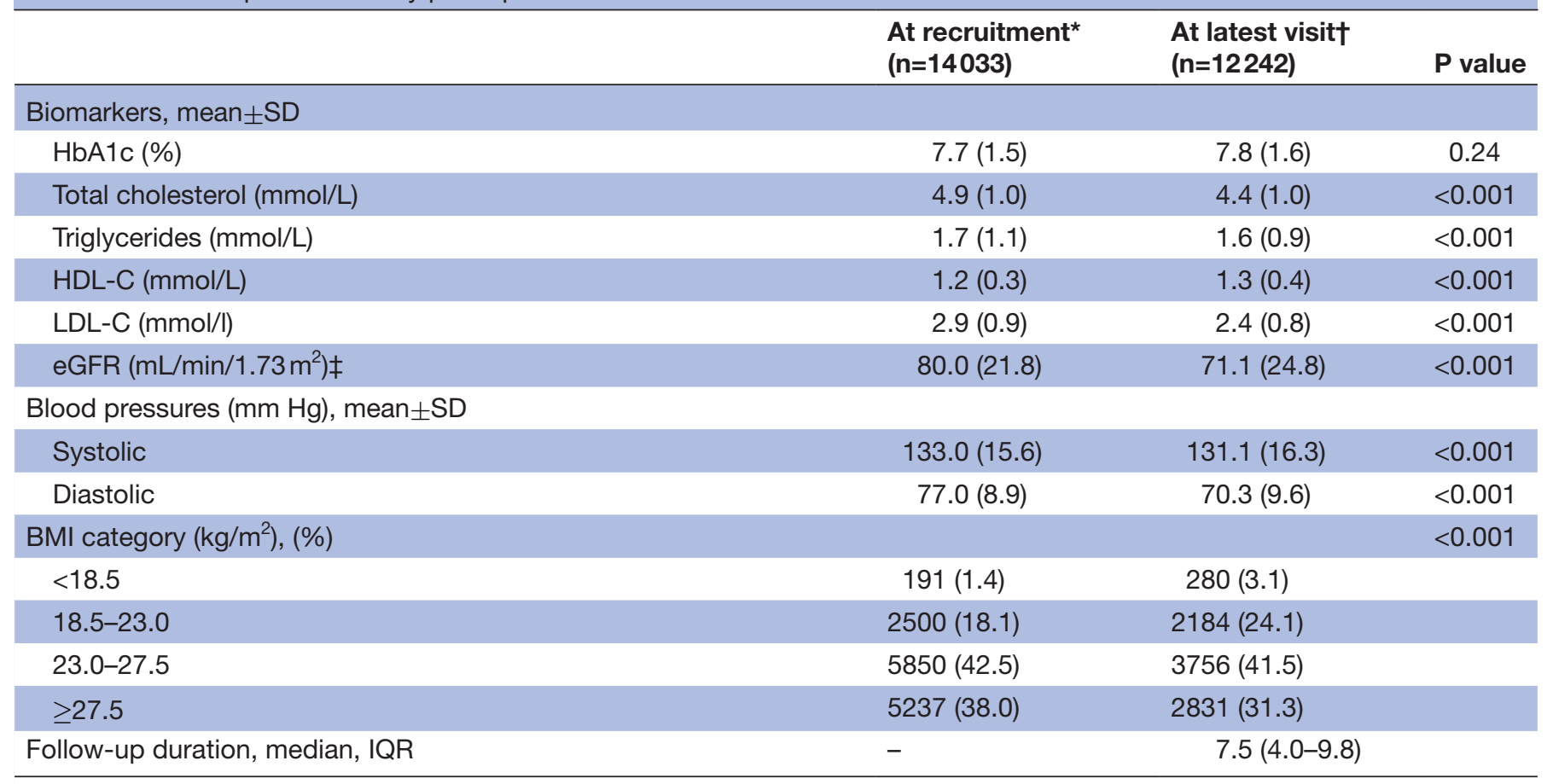

Numbers missing at recruitment: HbA1c $(n=1124)$, total cholesterol $(n=6656)$, triglycerides $(n=6659)$, HDL-C $(n=6671)$, LDL-C ( $n=6753)$, eGFR $(n=5426)$, blood pressure $(n=440), B M I(n=255)$. Numbers missing at latest visit: total cholesterol $(n=5042)$, triglycerides $(n=5040)$, HDL-C $(n=5040)$, LDL-C $(n=5041)$, eGFR $(n=5058)$, blood pressure $(n=194)$, BMI $(n=3191)$; most missing values are due to lack of records in extracted data.

*Variables measured within 1-year window from date of recruitment were used.

††date of the Latest Visit Was Defined Using the Date of the Last Hba1c Measurement for Each Subject, and Other Variables Measured Closest to This Date Within 1-year Window Were Used as the Latest Measurement. feGFR was calculated based on the CKD-EPI formula.

$\mathrm{BMI}$, body mass index; CKD-EPI, chronic kidney disease epidemiology collaboration; eGFR, estimated glomerular filtration rate; HbA1c, haemoglobin A1c; HDL-C, high-density lipoprotein cholesterol; LDL-C, low-density lipoprotein cholesterol.

The median duration of diabetes in the cohort was 7 years with an IQR of 3 to $14.68 .5 \%, 10.9 \%$ and $9.0 \%$ of participants reported a prior diagnosis of hypertension, diabetic retinopathy and diabetic kidney disease, respectively. Approximately half of the participants reported a family history of hypertension $(48.7 \%)$ and $76.6 \%$ reported a family history of diabetes. Participants who have been actively followed up were similar to the overall cohort at recruitment, except for slightly younger age and a greater proportion of those working (online supplementary table $1)$.

Disease characteristics of the study participants at recruitment and the latest visit in medical records are presented in table 4. Participants had a mean HbAlc of $7.7 \pm 1.5 \%$ at recruitment and similar levels were observed at their latest visit. There was a slight improvement in diastolic blood pressure (from $77 \pm 8.9 \mathrm{~mm} \mathrm{Hg}$ to $70 \pm 9.6$ $\mathrm{mm} \mathrm{Hg}$ ), and low-density lipoprotein cholesterol (from $2.9 \pm 0.9 \mathrm{mmol} / \mathrm{L}$ to $2.4 \pm 0.8 \mathrm{mmol} / \mathrm{L}$ ) between recruitment and last visit. The prevalence of obesity, defined as body mass index $\geq 27.5 \mathrm{~kg} / \mathrm{m}^{2}$ using Asian-specific cutoffs, ${ }^{22}$ decreased from $38.0 \%$ to $31.3 \%$ during this time. The median follow-up duration from recruitment to the last visit recorded was 7.5 years.

\section{Findings to date}

Data from the cohort have been used to identify determinants of diabetes and related complications. The longitudinal nature of the cohort and the linkage to a variety of records data have allowed the time trend analyses of diabetes control over time. An examination of determinants of poor glycaemic control in primary care over 5 years identified treatment with insulin at baseline, Malay or Indian ethnicity and presence of retinopathy to be associated with poor glycaemic control. ${ }^{23}$ The availability of serial HbAlc values has been used to examine patterns of longitudinal HbAlc control. Four distinct patterns were identified, the largest being a low-stable pattern with mean $\mathrm{HbAlc}$ of $7.1 \%$ over time, followed by a moderate stable pattern with mean $\mathrm{HbA1c}$ of $8.5 \%$, a pattern of deteriorating glycaemic control and one of improving glycaemic control. These patterns were associated with differential risks of late-stage complications and death. ${ }^{24}$ The role of diabetes treatment in shaping the HbA1c patterns has also been examined, with findings revealing that treatment by and large matched the extent of dysglycemia, and that HbA1c deterioration occurred in spite of treatment intensification and not due to a lack of intensification. ${ }^{25}$ 
Using biological samples collected from the cohort participants, we have also focused on identifying correlates and markers for diabetes and diabetic kidney disease. As part of the Asian Genetic Epidemiology Network Type 2 Diabetes Consortium, the DC has contributed to identification of T2D-associated genetic loci such as KCNQ1, ${ }^{26}$ East Asian-specific PAX $4^{27} 28$ and trans-ethnic SSR1-RREB1 and $A R L 15$ which have been implicated in regulation of fasting insulin and fasting glucose. ${ }^{29} \mathrm{DC}$ has also contributed cases to replication studies of novel T2D susceptibility loci identified first in European populations or other Asian populations ${ }^{30} 31$ as well as to transancestral investigations into the genetic architecture of diabetes. ${ }^{3233}$ More recently, whole exome sequencing analyses across multiple ancestries have identified modest rare-variant associations with T2D. ${ }^{20}{ }^{21}$ In addition to diabetes metaanalyses, the DC has also contributed to large-scale metaanalysis of diabetic kidney disease. ${ }^{34} 35$ Other analyses have demonstrated the significant associations of plasma tumor necrosis factor $\alpha$ and its receptors, ${ }^{17}$ pentosidine ${ }^{36}$ (an advanced glycation end product) and urinary excretion of nephrin ${ }^{37}$ with reduced kidney function. Metabolomic analysis of urine samples from DC participants through liquid chromatography-mass spectrometry and gas chromatography-mass spectrometry has identified several metabolites that could potentially serve as markers of non-proteinuric diabetic kidney disease. ${ }^{38}$

The complete list of publications based on the DC data is available online (https://blog.nus.edu.sg/sphs/ publications/).

\section{Strengths and limitations}

The main strengths of the DC are the focus on diabetes, which is one of the biggest public health challenges in this century, the relatively large pool of participants and a follow-up duration of more than a decade. The linkage of cohort data with medical records and disease registries is an important advantage for the cohort as this has allowed the in-depth and longitudinal tracking of several key clinical measures and outcomes in these patients. This linkage has also facilitated the capture of clinical data not only prospectively but also retrospectively before recruitment into the cohort. Another strength is the multiethnic composition of the cohort, representing three major ethnic groups in Asia, and thus allowing the evaluation of interethnic variation in diabetes progression, complication risk and outcomes. The stored biological samples are also an asset of the cohort, making it possible to examine novel and emerging biomarkers and genetic determinants in this population.

This study is not without its limitations. DC is a prevalence cohort, which recruited participants with varying durations of disease, and potentially at different stages of the natural history of disease. Another limitation is the low rate of active follow-up of participants, which has been substantially overcome through the linkage with medical records and disease registries. In spite of these limitations, the cohort continues to yield insights into diabetes and its complications in the Asian context.

\section{Collaboration}

We welcome potential collaboration with other researchers. Researchers can visit the Saw Swee Hock School of Public Health website (https://blog.nus.edu. $\mathrm{sg} / \mathrm{sphs} /$ ) for information on submitting a request for data and/or samples.

Contributors KV conceived the present manuscript and prepared the final version for the submission. ML drafted the manuscript. ML, LWLT and MKHN conducted the data analysis. RVD, EST, KSC, WET and DEJS established the cohort and provided intellectual inputs to the manuscript. XS critically revised the manuscript. All authors reviewed and approved the final version of the manuscript.

Funding The study was funded by grants from the National Medical Research Council (NMRC/0850/2004), Biomedical Research Council of A Star (BMRC/05/1/21/19/425), Ministry of Health, Singapore, National University of Singapore and National University Health System.

Competing interests None declared.

Patient and public involvement Patients and/or the public were not involved in the design, or conduct, or reporting, or dissemination plans of this research.

Patient consent for publication Not required.

Ethics approval Ethics approval for the DC was provided by the National University of Singapore Institutional Review Board (NUS IRB) and National Health Group Domain Specific Review Board (NHG DSRB).

Provenance and peer review Not commissioned; externally peer reviewed.

Data availability statement Data are available upon reasonable request. Researchers can visit the Saw Swee Hock School of Public Health website (https:// blog.nus.edu.sg/sphs/) for information on submitting a request for data and/or samples.

Open access This is an open access article distributed in accordance with the Creative Commons Attribution Non Commercial (CC BY-NC 4.0) license, which permits others to distribute, remix, adapt, build upon this work non-commercially, and license their derivative works on different terms, provided the original work is properly cited, appropriate credit is given, any changes made indicated, and the use is non-commercial. See: http://creativecommons.org/licenses/by-nc/4.0/.

ORCID iD

Kavita Venkataraman http://orcid.org/0000-0002-3139-6998

\section{REFERENCES}

1 Whiting DR, Guariguata L, Weil C, et al. IDF diabetes atlas: global estimates of the prevalence of diabetes for 2011 and 2030. Diabetes Res Clin Pract 2011;94:311-21.

2 IDF diabetes atlas eighth edition. International diabetes Federation: international diabetes Federation2017

3 Thomas MC, Cooper ME, Zimmet P. Changing epidemiology of type 2 diabetes mellitus and associated chronic kidney disease. Nat Rev Nephrol 2016;12:73-81.

4 Yau JWY, Rogers SL, Kawasaki R, et al. Global prevalence and major risk factors of diabetic retinopathy. Diabetes Care 2012;35:556-64.

5 Lazzarini PA, Pacella RE, Armstrong DG, et al. Diabetes-Related lower-extremity complications are a leading cause of the global burden of disability. Diabet Med 2018. doi:10.1111/dme.13680. [Epub ahead of print: 23 May 2018] (published Online First: 2018/05/24).

6 Harding JL, Pavkov ME, Magliano DJ, et al. Global trends in diabetes complications: a review of current evidence. Diabetologia 2019;62:3-16.

7 Kosiborod M, Gomes MB, Nicolucci A, et al. Vascular complications in patients with type 2 diabetes: prevalence and associated factors in 38 countries (the discover study program). Cardiovasc Diabetol 2018;17:150.

8 GBD 2016 Disease and Injury Incidence and Prevalence Collaborators. Global, regional, and national incidence, prevalence, and years lived with disability for 328 diseases and injuries for 195 
countries, 1990-2016: a systematic analysis for the global burden of disease study 2016. Lancet 2017;390:1211-59.

9 GBD 2016 Causes of Death Collaborators. Global, regional, and national age-sex specific mortality for 264 causes of death, 19802016: a systematic analysis for the global burden of disease study 2016. Lancet 2017;390:1151-210.

10 Venkataraman K, Wee HL, Leow MKS, et al. Associations between complications and health-related quality of life in individuals with diabetes. Clin Endocrinol 2013;78:865-73.

11 Venkataraman K, Khoo C, Wee HL, et al. Associations between disease awareness and health-related quality of life in a multi-ethnic Asian population. PLoS One 2014;9:e113802.

12 Bommer C, Heesemann E, Sagalova V, et al. The global economic burden of diabetes in adults aged $20-79$ years: a cost-of-illness study. Lancet Diabetes Endocrinol 2017;5:423-30.

13 Ma RCW, Chan JCN. Type 2 diabetes in East Asians: similarities and differences with populations in Europe and the United States. Ann N Y Acad Sci 2013;1281:64-91.

14 Unnikrishnan R, Gupta PK, Mohan V. Diabetes in South Asians: phenotype, clinical presentation, and natural history. Curr Diab Rep 2018;18:30.

15 National population health survey 2016/2017: Mininstry of health, Singapore, 2017. Available: https://www.moh.gov.sg/resourcesstatistics/reports/national-population-health-survey-2016-17 [Accessed 5th 2019].

16 Ang Y, Yap CW, Saxena N, et al. Diabetes-Related lower extremity amputations in Singapore. Proceedings of Singapore Healthcare 2017;26:76-80

17 Ng DPK, Fukushima M, Tai BC, et al. Reduced GFR and albuminuria in Chinese type 2 diabetes mellitus patients are both independently associated with activation of the TNF-alpha system. Diabetologia 2008;51:2318-24.

18 Tan KHX, Tan LWL, Sim X, et al. Cohort profile: the Singapore multiethnic cohort (mec) study. Int J Epidemiol 2018;47:699-699j.

19 Sim X, Ong RT-H, Suo C, et al. Transferability of type 2 diabetes implicated loci in multi-ethnic cohorts from Southeast Asia. PLoS Genet 2011;7:e1001363.

20 Flannick J, Mercader JM, Fuchsberger C, et al. Exome sequencing of 20,791 cases of type 2 diabetes and 24,440 controls. Nature 2019;570:71-6.

21 Fuchsberger C, Flannick J, Teslovich TM, et al. The genetic architecture of type 2 diabetes. Nature 2016;536:41-7.

22 WHO Expert Consultation. Appropriate body-mass index for Asian populations and its implications for policy and intervention strategies. Lancet 2004;363:157-63.

23 Tan NC, Barbier S, Lim WY, et al. 5-Year longitudinal study of determinants of glycemic control for multi-ethnic Asian patients with type 2 diabetes mellitus managed in primary care. Diabetes Res Clin Pract 2015;110:218-23.
24 Luo M, Lim WY, Tan CS, et al. Longitudinal trends in HbA1c and associations with comorbidity and all-cause mortality in Asian patients with type 2 diabetes: a cohort study. Diabetes Res Clin Pract 2017:133:69-77.

25 Luo M, Tan CS, Lim WY, et al. Association of diabetes treatment with long-term glycemic patterns in patients with type 2 diabetes mellitus: a prospective cohort study. Diabetes Metab Res Rev 2019;35:e3122.

26 Unoki H, Takahashi A, Kawaguchi T, et al. Snps in KCNQ1 are associated with susceptibility to type 2 diabetes in East Asian and European populations. Nat Genet 2008;40:1098-102.

27 Cho YS, Chen C-H, Hu C, et al. Meta-Analysis of genome-wide association studies identifies eight new loci for type 2 diabetes in East Asians. Nat Genet 2011;44:67-72.

$28 \mathrm{Ma} \mathrm{RCW}$, Hu C, Tam CH, et al. Genome-Wide association study in a Chinese population identifies a susceptibility locus for type 2 diabetes at 7q32 near Pax4. Diabetologia 2013;56:1291-305.

29 Scott RA, Lagou V, Welch RP, et al. Large-Scale association analyses identify new loci influencing glycemic traits and provide insight into the underlying biological pathways. Nat Genet 2012;44:991-1005.

30 Tan JT, Ng DPK, Nurbaya S, et al. Polymorphisms identified through genome-wide association studies and their associations with type 2 diabetes in Chinese, Malays, and Asian-Indians in Singapore. J Clin Endocrinol Metab 2010;95:390-7.

31 Dorajoo R, Blakemore AIF, Sim X, et al. Replication of 13 obesity loci among Singaporean Chinese, Malay and Asian-Indian populations. Int J Obes 2012;36:159-63.

32 Mahajan A, MJ G, Zhang W. Genome-Wide trans-ancestry metaanalysis provides insight into the genetic architecture of type 2 diabetes susceptibility. Nat Genet 2014;46:234-44.

33 Horikoshi M, Pasquali L, Wiltshire S, et al. Transancestral finemapping of four type 2 diabetes susceptibility loci highlights potential causal regulatory mechanisms. Hum Mol Genet 2016;25:2070-81.

34 Lim XL, Nurbaya S, Salim A, et al. Kcnq1 SNPs and susceptibility to diabetic nephropathy in East Asians with type 2 diabetes. Diabetologia 2012;55:2402-6.

35 van Zuydam NR, Ahlqvist E, Sandholm N, et al. A genome-wide association study of diabetic kidney disease in subjects with type 2 diabetes. Diabetes 2018;67:1414-27.

36 Lim X-L, Teo B-W, Tai B-C, et al. Pentosidine levels in nonproteinuric diabetes associated with both low estimated glomerular filtration rate and cataract. Diabetes Metab Syndr Obes 2012;5:155-64.

$37 \mathrm{Ng}$ DPK, Tai B-C, Tan E, et al. Nephrinuria associates with multiple renal traits in type 2 diabetes. Nephrol Dial Transplant 2011;26:2508-14.

$38 \mathrm{Ng}$ DPK, Salim A, Liu Y, et al. A metabolomic study of low estimated GFR in non-proteinuric type 2 diabetes mellitus. Diabetologia 2012;55:499-508. 